ISSN 2072-6643

www.mdpi.com/journal/nutrients

Review

\title{
Effect of Long-Chain Polyunsaturated Fatty Acid Supplementation on Neurodevelopmental Outcome in Full-Term Infants
}

\author{
Mijna Hadders-Algra \\ Department of Pediatrics-Developmental Neurology, University Medical Center Groningen, \\ Hanzeplein 1, 9713 GZ Groningen, The Netherlands; E-Mail: m.hadders-algra@med.umcg.nl; \\ Tel.:+31 50 3614247; Fax: +31503619158.
}

Received: 17 June 2010; in revised form: 6 July 2010 / Accepted: 15 July 2010 /

Published: 27 July 2010

\begin{abstract}
It takes more than 20 years before the human brain obtains its complex, adult configuration. Most dramatic developmental changes occur prenatally and early postnatally. During development, long-chain polyunsaturated fatty acids (LCPUFA) such as doxosahexaenoic acid (DHA) and arachidonic acid (AA) are accreted in the brain. Since breastfeeding is associated with a better developmental outcome than formula feeding, and human milk in contrast to traditional standard formula contains LCPUFA, the question arose whether LCPUFA supplementation of infant formula may promote the neurodevelopmental outcome. The current paper reviews the evidence available in full-term infants. It concludes that postnatal supplementation of formula with LCPUFA is associated with a beneficial effect on short-term neurodevelopmental outcome. However, no evidence is available that LCPUFA supplementation enhances neurodevelopmental outcome in full-term infants beyond the age of four months. Nevertheless, it should be realized that very limited information is available on the effect of LCPUFA supplementation on neurodevelopmental outcome at school age or later. It is conceivable that effects of LCPUFA supplementation first emerge or re-emerge at school age when more complex neural functions are expressed.
\end{abstract}

Keywords: LCPUFA; docosahexaenoic acid; arachidonic acid; full-term; infant; neurodevelopment; cognition; breast feeding 


\section{Introduction: Breastfeeding is Associated with Better Developmental Outcome}

More than 80 years ago, it had already been noted that breastfed infants had a better developmental outcome than infants fed other types of milk [1]. Now it is well established that breastfeeding is associated with a better neurological, cognitive and behavioral outcome than formula feeding [2-5]. However, it has not been clarified to which extent the composition of human milk explains this difference in development. For example, when perinatal and social confounders are taken into account, breastfeeding is associated with an increment of cognitive function of about 3 IQ points [4]. However, when the results are also adjusted for maternal IQ the cognitive benefit of breastfeeding is reduced to a small and non-significant difference of about 0.5 IQ points [6]. On the other hand, breastfeeding remained associated with a lower prevalence of fine manipulative dysfunction when maternal IQ was taken into account [3]. In addition, the finding that prolongation of the duration of breastfeeding - as a result of a cluster-randomized trial to promote breastfeeding - was associated with a better cognitive outcome at six years [7], suggests that the composition of human milk plays a role in the positive association between breastfeeding and outcome. Until recently, one of the differences between human milk and standard commercially available formulas was the presence of long-chain polyunsaturated fatty acids (LCPUFA) in human milk [8]. This fact inspired research on the effect of LCPUFA supplementation of formula in pre-term and full-term infants. The present paper aims at reviewing the effect of LCPUFA supplementation on neurodevelopmental outcome in full-term infants. The paragraphs reviewing the studies on the effect of LCPUFA supplementation are preceded by sections summarizing the ontogeny of the human brain and fatty acid accretion in the central nervous system.

\section{Ontogeny of the Human Brain}

The development of the human brain takes over 20 years. It is based on a continuous interaction between genetic information and environmental factors (for details on human brain ontogeny see [9-11]). Genetic instructions are, for instance, the major driving forces behind the functional topography of the human brain [12]. Environmental influences may vary from external sensory experiences, sensory experiences generated by self-produced motor activity and effects of chemical substances. The latter may be generated endogenously by the body or obtained from the external world, such as nutritional substances.

Brain development starts during the early phases of gestation with the proliferation of neurons in the germinal layers near the ventricles [13]. Next, neurons migrate in an orderly fashion to their final destinations. Meanwhile, they start to differentiate [14]. Neuronal differentiation includes the formation of dendrites and axons, the production of neurotransmitters and synapses, and the elaboration of the intracellular signaling machinery and the complex neural membranes [9,10]. The process of differentiation is particularly active in the few months prior to term age and the first few months post-term. The formation of synapses continues throughout life. Besides neural cells, glial cells are generated. The peak of glial cell production occurs in the second half of gestation [15]. Part of the glial cells take care of axonal myelination. Myelination takes place especially between the second trimester of gestation and the end of the first postnatal year. But it is first completed around the age of 40 years [16]. A remarkable feature of brain development is that it does not only consist of creation of components, but also of an elimination of elements. About half of the created neurons die off 
(apoptosis), in particular during mid-gestation [17]. Similarly, axons and synapses are eliminated, the latter especially between the onset of puberty and early adult age $[18,19]$. The shaping of the nervous system by these regressive phenomena is guided by neurochemical processes and neural activity [20]. The neural elements which fit the environment best persist, thus allowing for an adaptation of the brain to its own environment.

Brain development is characterized by phases of transition. A major transition occurs around three to four months post-term [21-23]. At three to four months, the subplate, the transient cortical structure mediating fetal and neonatal behavior, has been largely replaced by the cortical plate [22]. The cortical changes are paralleled by changes in behavior [21]. For instance, motor behavior changes from motility dominated by non-goal directed general movements into goal directed motility such as reaching behavior [24].

The continuous developmental changes in the brain have three practical implications for studies on the effect of LCPUFA supplementation. First, the effects of supplementation will depend on the timing of the supplementation. In other words, the effect of postnatal supplementation in pre-terms may differ from that in full-term infants. Second, the neurodevelopmental instruments to assess the effect of intervention should be age-specific. Third, the effects of supplementation on neurodevelopmental outcome in early infancy may differ from those on outcome at later age. Highly relevant for studies on the effect of nutritional intervention during early life is the finding that the development of neural dysfunction due to prenatal, perinatal or neonatal adversities may be characterized by phases in which dysfunction is not expressed. For instance, the Groningen Perinatal Project demonstrated that prenatal, perinatal and neonatal adversities were strongly associated with neonatal neurological morbidity. However, at the age of 18 months, the association between early risk factors and neurological dysfunction had disappeared to a large extent. It was first at school age, in particular from nine years onwards, that clear associations between early adversities and minor neurological dysfunction, learning and behavioral problems re-emerged [25].

\section{Fatty Acid Accretion in the Human Brain}

Fatty acids are major components of brain tissue: about 50 to $60 \%$ of dry adult brain weight consists of fatty acids [26]. Long-chain polyunsaturated fatty acids (LCPUFA), such as doxosahexaenoic acid

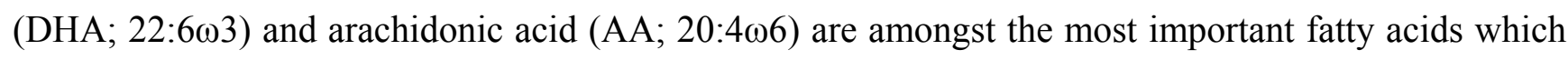
are incorporated into the brain, in particular into the synaptic membranes [26-28]. Initially LCPUFA accretion in human neural tissue proceeds rather slowly [29]. An LCPUFA accretion spurt first occurs in the last trimester of gestation [30,31]. There is some evidence that accretion of AA in the brain during the last trimester exceeds that of DHA, so that at term age the brain contains relatively more AA than DHA [30,31]. After term age, LCPUFA accretion in the nervous system continues. Gradually accretion of DHA surpasses that of AA. As a result, DHA is the major LCPUFA in the adult brain. In the brain LCPUFA accumulate mainly in the cortical gray matter, in particular in the synaptic membranes, and to a lesser extent in the white matter [27,28]. The baboon study of Diau et al. [27] indicated that highest concentrations of DHA and AA are found in the precentral, postcentral, prefrontal and occipital cortices, basal ganglia, hippocampus, thalamus and cerebellum, a finding which suggests that LCPUFA intake might affect in particular circuitries involved in sensorimotor integration, attention-executive function and memory. 
DHA and AA can be obtained directly from the diet or by endogeneous conversion of the parent

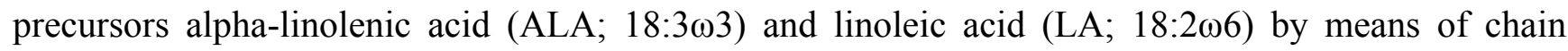
elongation and desaturation. The fetus and newborn infant are capable of these conversion processes, but the enzymatic systems involved seem to be unable to supply sufficient LCPUFA to meet the high requirements until 16 weeks after term age [28]. This means that during early development, LCPUFA supply is largely dependent on dietary intake of DHA and AA. For the fetus, this implies that it is dependent on maternal intake of LCPUFA, while the young infant has to rely on LCPUFA in milk $[32,33]$.

\section{LCPUFA Supplementation and Development in Full-Term Infants}

The systematic review of Simmer et al. [34] concluded that the RCTs on the effect of postnatal LCPUFA supplementation of formula milk in term infants did not show a beneficial effect of LCUPFA on neurodevelopmental outcome. However, this conclusion may be slightly modified when age at outcome is taken into account. The effect of LCPUFA supplementation on outcome in early infancy differs from that after the age of four months. This is illustrated in Table 1, which summarizes outcome until four months, and Table 2, which addresses outcome after the age of four months. The studies are rank ordered according to the level of DHA supplementation. Most studies evaluated visual development, cognitive or motor development. Table 1 shows that one of the three studies, in which formulae containing less than $0.30 \%$ DHA were evaluated, reported a better outcome in the supplemented infants, whereas seven out of eight studies in which formula with $\geq 0.30 \%$ DHA were used demonstrated a better developmental outcome in supplemented infants. On the basis of the limited number of studies, in particular those which studied the effect of relatively low DHA dosages, it can not be determined whether a beneficial effect of DHA supplementation on developmental outcome at early age depends on DHA dosage. Nevertheless, the 'meta-regression analysis' of Uauy et al. [35] suggested that the effect of postnatal LCPUFA supplementation in term infants on early visual development may be dependent on DHA dosage. It is also conceivable that a dosage of about $0.30 \%$ DHA may be sufficient to achieve the effect, as the recent study of Birch et al. [37] indicated that the effect of DHA on visual development was similar for supplementation with $0.32 \%$, $0.64 \%$ or $0.96 \%$ DHA. Table 2 indicates, however, that the positive effect of postnatal LCPUFA supplementation on developmental outcome could not be demonstrated for outcome after the age of four months. 
Table 1. LCPUFA supplementation in term infants and outcome until four months of age (adapted and updated from Hadders-Algra 2005 [36]).

\begin{tabular}{|c|c|c|c|c|c|c|c|c|}
\hline Author(s) & Groups & $\begin{array}{l}\text { Duration of } \\
\text { supplementation }\end{array}$ & DHA content & AA content & $\begin{array}{l}\text { Age at FU } \\
\text { (months) }\end{array}$ & $\begin{array}{l}\text { Attrition at } \\
\text { last FU }\end{array}$ & $\begin{array}{l}\text { Assessment at } \\
\text { follow-up }\end{array}$ & Results \\
\hline $\begin{array}{l}\text { Carlson et al. } \\
1996[37]\end{array}$ & $\begin{array}{ll}\text { E } & n=19 \\
C & n=20 \\
\text { BF } & n=19\end{array}$ & $\begin{array}{l}? \\
\mathrm{BF} \geq 3 \mathrm{mo}\end{array}$ & $0.10 \%$ & $0.43 \%$ & 2 and 4 & $38 \%$ & $\begin{array}{l}\text { Teller visual acuity } \\
{[38]}\end{array}$ & $\begin{array}{l}2 \text { mo: } \mathrm{E}>\mathrm{C} ; \mathrm{BF}>\mathrm{C} \\
4 \mathrm{mo}: \mathrm{E}=\mathrm{C} ; \mathrm{BF}=\mathrm{C}\end{array}$ \\
\hline $\begin{array}{l}\text { Auestad et al. } \\
1997[39]^{\text {a }}\end{array}$ & $\begin{array}{ll}\mathrm{E}_{1} & \mathrm{n}=26 \\
\mathrm{E}_{2} & \mathrm{n}=28 \\
\mathrm{C} & \mathrm{n}=28 \\
\mathrm{BF} & \mathrm{n}=38 \\
\end{array}$ & $\begin{array}{l}\geq 4 \mathrm{mo} \\
\mathrm{BF} \geq 4 \mathrm{mo}\end{array}$ & $\begin{array}{l}0.12 \% \\
0.20 \%\end{array}$ & $0.43 \%$ & 2 and 4 & $\begin{array}{l}39 \% \\
\text { (at } 12 \mathrm{mo} \text { ) }\end{array}$ & $\begin{array}{l}\text { FPL [40] } \\
\text { Sweep VEP [41] }\end{array}$ & $\begin{array}{l}E_{1}=C ; E_{2}=C \\
B F=C\end{array}$ \\
\hline $\begin{array}{l}\text { Auestad et al. } \\
2001[42]\end{array}$ & $\begin{array}{ll}\mathrm{E}_{1} & \mathrm{n}=58 \\
\mathrm{E}_{2} & \mathrm{n}=60 \\
\mathrm{C} & \mathrm{n}=56 \\
\mathrm{BF} & \mathrm{n}=120\end{array}$ & $\begin{array}{l}12 \mathrm{mo} \\
\mathrm{BF} \geq 3 \mathrm{mo}\end{array}$ & $\begin{array}{l}0.14 \% \text { (egg) } \\
0.13 \% \\
\text { (fish/fungal) }\end{array}$ & $\begin{array}{l}0.45 \% \\
0.46 \%\end{array}$ & 1,2 and 4 & $\begin{array}{l}27 \% \\
\text { (at } 12 \mathrm{mo} \text { ) }\end{array}$ & $\begin{array}{l}\text { Teller visual acuity } \\
{[38]}\end{array}$ & $\begin{array}{l}\mathrm{E}_{1}=\mathrm{C} ; \mathrm{E}_{2}=\mathrm{C} \\
\mathrm{BF}=\mathrm{C}\end{array}$ \\
\hline $\begin{array}{l}\text { Agostoni et al. } \\
1995 \text { [43] }\end{array}$ & $\begin{array}{ll}\mathrm{E} & \mathrm{n}=27 \\
\mathrm{C} & \mathrm{n}=29 \\
\mathrm{BF} & \mathrm{n}=30\end{array}$ & $\begin{array}{l}4 \mathrm{mo} \\
\mathrm{BF} \geq 4 \mathrm{mo}\end{array}$ & $0.30 \%$ & $0.44 \%$ & 4 & $4 \%$ & $\begin{array}{l}\text { Brunet - Lezine DQ } \\
\text { [44] }\end{array}$ & $\begin{array}{l}\mathrm{E}>\mathrm{C} \\
\mathrm{BF}>\mathrm{C}\end{array}$ \\
\hline $\begin{array}{l}\text { Bouwstra et al. } \\
2003[45]^{\mathrm{b}}\end{array}$ & $\begin{array}{ll}\mathrm{E} & \mathrm{n}=131 \\
\mathrm{C} & \mathrm{n}=119 \\
\mathrm{BF} & \mathrm{n}=147\end{array}$ & $\begin{array}{l}2 \mathrm{mo} \\
\mathrm{BF} \text { variable; } \\
\text { median } 9 \mathrm{wk}\end{array}$ & $0.30 \%$ & $0.45 \%$ & 3 & $16 \%$ & $\begin{array}{l}\text { Quality of general } \\
\text { movements [46] }\end{array}$ & $\begin{array}{l}\mathrm{E}>\mathrm{C} \\
\mathrm{BF}>\mathrm{C}\end{array}$ \\
\hline $\begin{array}{l}\text { Makrides et al. } \\
2000[47]\end{array}$ & $\begin{array}{ll}\mathrm{E}_{1} & \mathrm{n}=24 \\
\mathrm{E}_{2} & \mathrm{n}=23 \\
\mathrm{C} & \mathrm{n}=21 \\
\mathrm{BF} & \mathrm{n}=46 \\
\end{array}$ & $\begin{array}{l}12 \mathrm{mo} \\
\mathrm{BF} \geq 3 \mathrm{mo}\end{array}$ & $\begin{array}{l}0.34 \% \\
0.35 \%\end{array}$ & $0.34 \%$ & 4 & $\begin{array}{l}18 \% \\
\text { (at } 8 \mathrm{mo})\end{array}$ & VEP [41] & $\begin{array}{l}\mathrm{E}_{1}=\mathrm{C} ; \mathrm{E}_{2}=\mathrm{C} \\
\mathrm{BF}=\mathrm{C}\end{array}$ \\
\hline $\begin{array}{l}\text { Makrides et al. } \\
1995 \text { [48] }\end{array}$ & $\begin{array}{ll}\mathrm{E} & \mathrm{n}=13 \\
\mathrm{C} & \mathrm{n}=19 \\
\mathrm{BF} & \mathrm{n}=23\end{array}$ & $\begin{array}{l}? \\
\mathrm{BF} \geq 4 \mathrm{mo}\end{array}$ & $0.36 \%$ & & 4 & $11 \%$ & VEP [41] & $\begin{array}{l}\mathrm{E}>\mathrm{C} \\
\mathrm{BF}>\mathrm{C}\end{array}$ \\
\hline
\end{tabular}


Table 1. Cont.

\begin{tabular}{|c|c|c|c|c|c|c|c|c|}
\hline $\begin{array}{l}\text { Birch et al. } \\
1998 \text { [49] }\end{array}$ & $\begin{array}{ll}\mathrm{E}_{1} & \mathrm{n}=23 \\
\mathrm{E}_{2} & \mathrm{n}=22 \\
\mathrm{C} & \mathrm{n}=23 \\
\mathrm{BF} & \mathrm{n}=21\end{array}$ & $\begin{array}{l}4 \mathrm{mo} \\
\mathrm{BF} \geq 4 \mathrm{mo}\end{array}$ & $\begin{array}{l}0.36 \% \\
0.35 \%\end{array}$ & $0.72 \%$ & 1.5 and 4 & $18 \%$ & $\begin{array}{l}\text { FPL [40] } \\
\text { Sweep VEP [41] }\end{array}$ & $\begin{array}{l}\mathrm{E}=\mathrm{C} ; \mathrm{BF}=\mathrm{C} \\
\mathrm{E}_{1}>\mathrm{C} ; \mathrm{E}_{2}>C \\
\mathrm{BF}>\mathrm{C}\end{array}$ \\
\hline $\begin{array}{l}\text { Birch et al. } \\
2005 \text { [50] }\end{array}$ & $\begin{array}{ll}\mathrm{E} & \mathrm{n}=46 \\
\mathrm{C} & \mathrm{n}=46\end{array}$ & $12 \mathrm{mo}$ & $0.36 \%$ & $0.72 \%$ & $\begin{array}{l}1.5 \text { and } 4 \\
4\end{array}$ & $11 \%$ & $\begin{array}{l}\text { Sweep VEP [41] } \\
\text { Stereoacuity [51] }\end{array}$ & $\begin{array}{l}E>C \\
E>C\end{array}$ \\
\hline $\begin{array}{l}\text { Ünay et al. } \\
2004 \text { [52] }\end{array}$ & $\begin{array}{ll}\mathrm{E} & \mathrm{n}=22 \\
\mathrm{C} & \mathrm{n}=22 \\
\mathrm{BF} & \mathrm{n}=23\end{array}$ & $\begin{array}{l}4 \mathrm{mo} \\
\mathrm{BF} \geq 4 \mathrm{mo}\end{array}$ & $0.50 \%$ & & 4 & $16 \%$ & BAEP [53] & $\begin{array}{l}\mathrm{E}>\mathrm{C} \\
\mathrm{BF}>\mathrm{C}\end{array}$ \\
\hline $\begin{array}{l}\text { Birch et al. } \\
2010^{*} \text { [54] }\end{array}$ & $\begin{array}{ll}E_{1} & n=74 \\
E_{2} & n=75 \\
E_{3} & n=75 \\
C & n=76 \\
\end{array}$ & $12 \mathrm{mo}$ & $\begin{array}{l}0.32 \% \\
0.64 \% \\
0.96 \%\end{array}$ & $0.64 \%$ & 1.5 and 4 & $12 \%$ & Sweep VEP [41] & $\mathrm{E}_{1}=\mathrm{E}_{2}=\mathrm{E}_{3}>\mathrm{C}$ \\
\hline
\end{tabular}


$\mathrm{E}=$ experimental group, $\mathrm{FPL}=$ forced preference looking, $\mathrm{FU}=$ follow-up, $\mathrm{mo}=$ months, $\mathrm{VEP}=$ visual evoked potential.

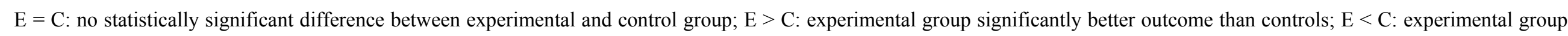
significantly worse than control group.

Superscript letters in authors column indicate that studies evaluated outcome in more or less the same study groups.

* Birch et al. 2010: no exact numbers on assessed infants at each age available.

Table 2. LCPUFA supplementation in term infants and outcome beyond 4 months (adapted and updated from Hadders-Algra 2005 [26]).

\begin{tabular}{|c|c|c|c|c|c|c|c|c|}
\hline Author(s) & Groups & $\begin{array}{l}\text { Duration of } \\
\text { supplementation }\end{array}$ & DHA content & AA content & $\begin{array}{l}\text { Age at FU } \\
\text { (months) }\end{array}$ & $\begin{array}{l}\text { Attrition at } \\
\text { last FU }\end{array}$ & $\begin{array}{l}\text { Assessment at } \\
\text { follow-up }\end{array}$ & Results \\
\hline $\begin{array}{l}\text { Carlson et al. } \\
1996[37]\end{array}$ & $\begin{array}{ll}\mathrm{E} & \mathrm{n}=19 \\
\mathrm{C} & \mathrm{n}=20 \\
\mathrm{BF} & \mathrm{n}=19\end{array}$ & $\begin{array}{l}? \\
\mathrm{BF} \geq 3 \mathrm{mo}\end{array}$ & $0.10 \%$ & $0.43 \%$ & 6 and 12 & $\geq 38 \%$ & $\begin{array}{l}\text { Teller visual acuity } \\
{[38]}\end{array}$ & $\begin{array}{l}\mathrm{E}=\mathrm{C} \\
\mathrm{BF}=\mathrm{C}\end{array}$ \\
\hline
\end{tabular}


Table 2. Cont.

\begin{tabular}{|c|c|c|c|c|c|c|c|c|}
\hline $\begin{array}{l}\text { Auestad et al. } \\
1997 \text { [39 }^{\mathrm{a}}\end{array}$ & $\begin{array}{ll}\mathrm{E}_{1} & \mathrm{n}=26 \\
\mathrm{E}_{2} & \mathrm{n}=28 \\
\mathrm{C} & \mathrm{n}=28 \\
\mathrm{BF} & \mathrm{n}=38 \\
\end{array}$ & $\begin{array}{l}\geq 4 \mathrm{mo} \\
\mathrm{BF} \geq 4 \mathrm{mo}\end{array}$ & $\begin{array}{l}0.12 \% \\
0.20 \%\end{array}$ & $0.43 \%$ & 6,9 and 12 & $39 \%$ & $\begin{array}{l}\text { FPL [40] } \\
\text { Sweep VEP [41] }\end{array}$ & $\begin{array}{l}\mathrm{E}=\mathrm{C} \\
\mathrm{BF}=\mathrm{C}\end{array}$ \\
\hline $\begin{array}{l}\text { Scott et al. } \\
1998[55]^{\text {a }}\end{array}$ & $\begin{array}{ll}\mathrm{E}_{1} & \mathrm{n}=38 \\
\mathrm{E}_{2} & \mathrm{n}=33 \\
\mathrm{C} & \mathrm{n}=42 \\
\mathrm{BF} & \mathrm{n}=60\end{array}$ & $\begin{array}{l}\geq 4 \mathrm{mo} \\
\mathrm{BF} \geq 3 \mathrm{mo}\end{array}$ & $\begin{array}{l}0.12 \% \\
0.20 \%\end{array}$ & $0.43 \%$ & $\begin{array}{l}12 \\
14\end{array}$ & $37 \%$ & $\begin{array}{l}\text { Bayley PDI / MDI } \\
{[56]} \\
\text { McArthur language [57] }\end{array}$ & $\begin{array}{l}\mathrm{E}_{1}=\mathrm{C} ; \mathrm{E}_{2}=\mathrm{C} ; \mathrm{BF}=\mathrm{C} \\
\mathrm{E}_{1}=\mathrm{C} ; \mathrm{E}_{2}<\mathrm{C} ; \mathrm{BF}=\mathrm{C}\end{array}$ \\
\hline $\begin{array}{l}\text { Auestad et al. } \\
2001 \text { [42] }\end{array}$ & $\begin{array}{ll}\mathrm{E}_{1} & \mathrm{n}=58 \\
\mathrm{E}_{2} & \mathrm{n}=60 \\
\mathrm{C} & \mathrm{n}=56 \\
\mathrm{BF} & \mathrm{n}=120\end{array}$ & $\begin{array}{l}12 \mathrm{mo} \\
\mathrm{BF} \geq 3 \mathrm{mo}\end{array}$ & $\begin{array}{l}0.14 \% \text { (egg) } \\
0.13 \% \\
\text { (fish/fungal) }\end{array}$ & $\begin{array}{l}0.45 \% \\
0.46 \%\end{array}$ & $\begin{array}{l}6,9,12 \\
6 \text { and } 9 \\
6 \text { and } 12 \\
6 \text { and } 12 \\
9 \text { and } 14\end{array}$ & $\begin{array}{l}27 \% \\
\text { (at } 12 \mathrm{mo} \text { ) }\end{array}$ & $\begin{array}{l}\text { Teller vis. acuity [38] } \\
\text { Fagan [58] } \\
\text { Bayley PDI / MDI } \\
\text { [56] } \\
\text { IBQ [59] } \\
\text { Language [57] }\end{array}$ & $\begin{array}{l}E=C ; B F=C \\
E=C ; B F=C \\
E=C ; B F=C \\
E=C ; B F=C \\
E=C ; B F=C\end{array}$ \\
\hline $\begin{array}{l}\text { Willats et al. } \\
1998[60]\end{array}$ & $\begin{array}{ll}\mathrm{E} & \mathrm{n}=21 \\
\mathrm{C} & \mathrm{n}=23 \\
\end{array}$ & $4 \mathrm{mo}$ & 0.20 & 0.30 & 10 & $38 \%$ & Problem solving task [60] & $\mathrm{E}>\mathrm{C}$ \\
\hline $\begin{array}{l}\text { Auestad et al. } \\
2003[61]^{\mathrm{a}}\end{array}$ & $\begin{array}{ll}E_{1} & n=35 \\
E_{2} & n=35 \\
C & n=37 \\
B F & n=50\end{array}$ & $\begin{array}{l}12 \mathrm{mo} \\
\mathrm{BF} \geq 3 \mathrm{mo}\end{array}$ & $\begin{array}{l}0.12 \% \\
0.23 \%\end{array}$ & $0.43 \%$ & 39 & $47 \%$ & $\begin{array}{l}\text { Teller vis. acuity } \\
\text { Beery VMI [62] } \\
\text { Stanford-Binet IQ [63] } \\
\text { Language [57] }\end{array}$ & $\begin{array}{l}\mathrm{E}=\mathrm{C} ; \mathrm{BF}=\mathrm{C} \\
\mathrm{E}=\mathrm{C} ; \mathrm{BF}=\mathrm{C} \\
\mathrm{E}=\mathrm{C} ; \mathrm{BF}=\mathrm{C} \\
\mathrm{E}=\mathrm{C} ; \mathrm{BF}=\mathrm{C}\end{array}$ \\
\hline $\begin{array}{l}\text { Bouwstra et al. } \\
2005[64]^{\mathrm{b}}\end{array}$ & $\begin{array}{ll}\text { E } & n=135 \\
C & n=157 \\
\text { BF } & n=154\end{array}$ & $\begin{array}{l}2 \mathrm{mo} \\
\mathrm{BF} \text { variable; } \\
\text { median } 9 \mathrm{wk}\end{array}$ & $0.30 \%$ & $0.45 \%$ & 18 & $6 \%$ & $\begin{array}{l}\text { Hempel neurolog exam } \\
{[65]} \\
\text { Bayley PDI / MDI } \\
{[56]}\end{array}$ & $\mathrm{E}=\mathrm{C} ; \mathrm{BF}=\mathrm{C}$ \\
\hline
\end{tabular}


Table 2. Cont.

\begin{tabular}{|c|c|c|c|c|c|c|c|c|}
\hline $\begin{array}{l}\text { De Jong et al. } \\
2010[3]^{\mathrm{b}}\end{array}$ & $\begin{array}{ll}\mathrm{E} & \mathrm{n}=91 \\
\mathrm{C} & \mathrm{n}=123 \\
\mathrm{BF} & \mathrm{n}=127\end{array}$ & $\begin{array}{l}2 \mathrm{mo} \\
\text { BF variable; } \\
\text { median } 9 \mathrm{wk}\end{array}$ & $0.30 \%$ & $0.45 \%$ & $9 \mathrm{yr}$ & $28 \%$ & $\begin{array}{l}\text { Touwen neurological } \\
\text { exam } \\
{[66]}\end{array}$ & $\mathrm{E}=\mathrm{C} ; \mathrm{BF}>\mathrm{C}$ \\
\hline $\begin{array}{l}\text { Agostoni et al. } \\
1997 \text { [67] }\end{array}$ & $\begin{array}{ll}\text { E } & \mathrm{n}=26 \\
\mathrm{C} & \mathrm{n}=30 \\
\mathrm{BF} & \mathrm{n}=25\end{array}$ & $\begin{array}{l}4 \mathrm{mo} \\
\mathrm{BF} \geq 4 \mathrm{mo}\end{array}$ & $0.30 \%$ & $0.44 \%$ & 24 & $10 \%$ & $\begin{array}{l}\text { Brunet - Lezine DQ } \\
{[44]}\end{array}$ & $\mathrm{E}=\mathrm{C} ; \mathrm{BF}=\mathrm{C}$ \\
\hline $\begin{array}{l}\text { Lucas et al. } \\
1999[68]\end{array}$ & $\begin{array}{l}\mathrm{E} \quad \mathrm{n}=125 \\
\mathrm{C} \mathrm{n}=125 \\
\mathrm{BF} \mathrm{n}=104\end{array}$ & $\begin{array}{l}6 \mathrm{mo} \\
\mathrm{BF} \geq 6 \mathrm{wk}\end{array}$ & $0.32 \%$ & $0.30 \%$ & $\begin{array}{l}9 \\
18 \\
\end{array}$ & $21 \%$ & $\begin{array}{l}\text { Knobloch DQ [69] } \\
\text { Bayley PDI / MDI } \\
{[56]}\end{array}$ & $\begin{array}{l}\mathrm{E}=\mathrm{C} ; \mathrm{BF}=\mathrm{C} \\
\mathrm{E}=\mathrm{C} ; \mathrm{BF}=\mathrm{C}\end{array}$ \\
\hline $\begin{array}{l}\text { Singhal et al. } \\
2007 \text { [70] }\end{array}$ & $\begin{array}{l}\text { E } \mathrm{n}=89 \\
\mathrm{C} \mathrm{n}=81 \\
\mathrm{BF} \mathrm{n}=73\end{array}$ & $\begin{array}{l}6 \mathrm{mo} \\
\mathrm{BF} \geq 6 \mathrm{wk}\end{array}$ & $0.32 \%$ & $0.30 \%$ & $\begin{array}{l}4-6 \mathrm{yr} \\
4-6 \mathrm{yr} \\
\end{array}$ & $46 \%$ & $\begin{array}{l}\text { Visual acuity [71] } \\
\text { Stereoacuity [51] }\end{array}$ & $\begin{array}{l}\mathrm{E}=\mathrm{C} ; \mathrm{BF}=\mathrm{C} \\
\mathrm{E}=\mathrm{C} ; \mathrm{BF}>\mathrm{C}\end{array}$ \\
\hline $\begin{array}{l}\text { Makrides et al. } \\
2000 \text { [47] }\end{array}$ & $\begin{array}{ll}\mathrm{E}_{1} & \mathrm{n}=19 \\
\mathrm{E}_{2} & \mathrm{n}=22 \\
\mathrm{C} & \mathrm{n}=19 \\
\mathrm{BF} & \mathrm{n}=23\end{array}$ & $\begin{array}{l}12 \mathrm{mo} \\
\mathrm{BF} \geq 3 \mathrm{mo}\end{array}$ & $\begin{array}{l}0.34 \% \\
0.35 \%\end{array}$ & $0.34 \%$ & $\begin{array}{l}8 \\
12 \text { and } 24\end{array}$ & $12 \%$ & $\begin{array}{l}\text { VEP [41] } \\
\text { Bayley PDI / MDI } \\
{[56]}\end{array}$ & $\begin{array}{l}E=C ; B F=C \\
\text { PDI: } E=C ; B F=C \\
12 \text { mo MDI: } E=C ; B F=C ; \\
24 \text { mo MDI: } E=C ; B F>C\end{array}$ \\
\hline $\begin{array}{l}\text { Birch et al. } \\
1998 \text { [49] }\end{array}$ & $\begin{array}{ll}\mathrm{E}_{1} & \mathrm{n}=19 \\
\mathrm{E}_{2} & \mathrm{n}=22 \\
\mathrm{C} & \mathrm{n}=20 \\
\mathrm{BF} & \mathrm{n}=46 \\
\end{array}$ & $\begin{array}{l}4 \mathrm{mo} \\
\mathrm{BF} \geq 4 \mathrm{mo} \\
\end{array}$ & $\begin{array}{l}0.36 \% \\
0.35 \%\end{array}$ & $0.72 \%$ & 6 and 12 & $26 \%$ & $\begin{array}{l}\text { FPL [40] } \\
\text { Sweep VEP [41] }\end{array}$ & $\begin{array}{l}\mathrm{E}=\mathrm{C} ; \mathrm{BF}=\mathrm{C} \\
6 \text { mo VEP: } \mathrm{E}=\mathrm{C} ; \\
\mathrm{BF}=\mathrm{C} ; 12 \text { mo VEP: } \\
\mathrm{E}_{1}>\mathrm{C} ; \mathrm{E}_{2}>\mathrm{C} ; \mathrm{BF}>\mathrm{C}\end{array}$ \\
\hline $\begin{array}{l}\text { Birch et al. } \\
2000[72]\end{array}$ & $\begin{array}{ll}\mathrm{E}_{1} & \mathrm{n}=19 \\
\mathrm{E}_{2} & \mathrm{n}=17 \\
\mathrm{C} & \mathrm{n}=20 \\
\end{array}$ & 4 mo & $\begin{array}{l}0.36 \% \\
0.35 \%\end{array}$ & $0.72 \%$ & 18 & $29 \%$ & $\begin{array}{l}\text { Bayley PDI / MDI } \\
{[56]}\end{array}$ & $\begin{array}{l}\text { MDI: } \mathrm{E}_{1}>\mathrm{C}, \mathrm{E}_{2}=\mathrm{C} \\
\text { PDI: } \mathrm{E}=\mathrm{C}\end{array}$ \\
\hline $\begin{array}{l}\text { Birch et al. } \\
2007[50]\end{array}$ & $\begin{array}{ll}\mathrm{E}_{1} & \mathrm{n}=19 \\
\mathrm{E}_{2} & \mathrm{n}=17 \\
\mathrm{C} & \mathrm{n}=20 \\
\mathrm{BF} & \mathrm{n}=32 \\
\end{array}$ & 4 mo & $\begin{array}{l}0.36 \% \\
0.35 \%\end{array}$ & $0.72 \%$ & $4 \mathrm{yr}$ & $34 \%$ & $\begin{array}{l}\text { visual acuity [74] } \\
\text { WPPSI [75] }\end{array}$ & $\begin{array}{l}\mathrm{E}_{1}=\mathrm{C}, \mathrm{E}_{2}>\mathrm{C}, \mathrm{BF}>\mathrm{C} \\
\text { VIQ: } \mathrm{E}=\mathrm{C}, \mathrm{BF}>\mathrm{C}, \mathrm{E}_{1} \\
\text { PIQ: } \mathrm{E}=\mathrm{C}, \mathrm{BF}=\mathrm{C}\end{array}$ \\
\hline
\end{tabular}


Table 2. Cont.

\begin{tabular}{|c|c|c|c|c|c|c|c|c|}
\hline $\begin{array}{l}\text { Birch et al. } \\
2005[35]\end{array}$ & $\begin{array}{ll}\mathrm{E} & \mathrm{n}=42 \\
\mathrm{C} & \mathrm{n}=44\end{array}$ & $12 \mathrm{mo}$ & $0.36 \%$ & $0.72 \%$ & $\begin{array}{l}9 \text { and } 12 \\
9 \text { and } 12\end{array}$ & $17 \%$ & $\begin{array}{l}\text { Sweep VEP [41] } \\
\text { Stereoacuity [51] }\end{array}$ & $\begin{array}{l}E>C \\
E=C\end{array}$ \\
\hline $\begin{array}{l}\text { Birch et al. } \\
2010^{*}[54]\end{array}$ & $\begin{array}{ll}E_{1} & n=64 \\
E_{2} & n=59 \\
E_{3} & n=65 \\
C & n=56\end{array}$ & $12 \mathrm{mo}$ & $\begin{array}{l}0.32 \% \\
0.64 \% \\
0.96 \%\end{array}$ & $0.64 \%$ & 9 and 12 & $28 \%$ & Sweep VEP [41] & $\mathrm{E}_{1}=\mathrm{E}_{2}=\mathrm{E}_{3}>\mathrm{C}$ \\
\hline
\end{tabular}

Note that some of the studies of Table 2 are also included in Table 1; this implies that the groups were re-assessed after the age of four months.

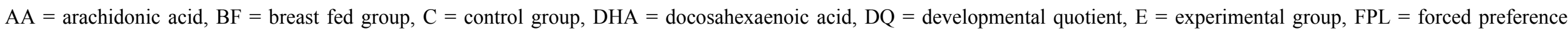

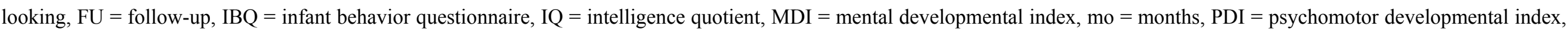
$\mathrm{VEP}=$ visual evoked potential, VMI = visuomotor integration, WPPSI = Wechsler Preschool and Primary Scale of Intelligence

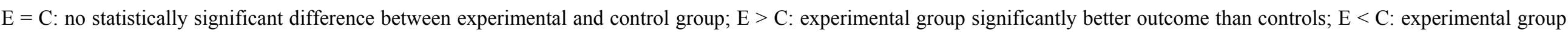
significantly worse than control group.

Superscript letters in authors column indicate that studies evaluated outcome in more or less the same study groups.

* Birch et al. 2010: no exact numbers on assessed infants at each age available. 


\section{Concluding Remarks}

Postnatal supplementation of formula with LCPUFA in full-term infants is associated with a beneficial effect on short term neurodevelopmental outcome. Possibly, this short term beneficial effect is brought about by DHA levels of at least $0.30 \%$. Interestingly, this putative threshold level is similar to the average level of DHA in human milk worldwide $(0.32 \%$, [8]). No long-term beneficial effects of LCPUFA supplementation in full-term infants have been demonstrated. However, it should be realized that very limited information is available on the effect of LCPUFA supplementation on neurodevelopmental outcome at school age or later. It is conceivable that effects of LCPUFA supplementation first emerge or re-emerge at school age when more complex neural functions are expressed. The latter possibility may be illustrated by the age-dependency of developmental differences between breastfed and formula-fed infants in the Netherlands. At the age of three months breast-fed infants have a better neurological condition than formula-fed infants [45], at 18 months this difference has disappeared [64], to re-emerge at the age of at least $3 \frac{1}{2} 2$ years $[3,76]$

\section{Acknowledgements}

The author's work on LCPUFA is supported by Early Nutrition Programming Project (EARNEST), which is funded under the Food Quality and Safety Priority of the Sixth Framework Programme for Research and Technical Development of the European Community (FOOD-CT-2005-007036, www.metabolic-programming.org).

\section{References}

1. Hoefer, C.; Hardy, M.C. Later development of breast fed and artificially fed infants. J. Am. Med. Assoc. 1929, 92, 615-620.

2. Lanting, C.I.; Fidler, V.; Huisman, M.; Touwen, B.C.; Boersma, E.R. Neurological differences between 9-year-old children fed breast-milk or formula-milk as babies. Lancet 1994, 344, 1319-1322.

3. De Jong, C.; Kikkert, H.K.; Fidler, V.; Hadders-Algra, M. The Groningen LCPUFA study: no effect of postnatal long-chain polyunsaturated fatty acids in healthy term infants on neurological condition at 9 years. Br. J. Nutr. 2010, Epub ahead of print.

4. Anderson, J.W.; Johnstone, B.M.; Remley, D.T. Breast-feeding and cognitive development: a meta-analysis. Am. J. Clin. Nutr. 1999, 70, 525-535.

5. Julvez, J.; Ribas-Fitó, N.; Forns, M.; Garcia-Esteban, R.; Torrent, M.; Sunyer, J. Attention behaviour and hyperactivity at age 4 and duration of breast-feeding. Acta Paediatr. 2007, 96, 842-847.

6. Der, G.; Batty, G.D.; Deary, I.J. Effect of breast feeding on intelligence in children: prospective sibling pairs analysis, and meta-analysis. B.M.J. 2006, 333, 945. 
7. Kramer, M.S.; Aboud, F.; Mironova, E.; Vanilovich, I.; Platt, R.W.; Matush, L.; Igumnov, S.; Fombonne, E.; Bogdanovich, N.; Ducruet, T.; Collet, J.P.; Chalmers, B.; Hodnett, E.; Davidovsky, S.; Skugarevsky, O.; Trofimovich, O.; Kozlova, L.; Shapiro, S.; Promotion of Breastfeeding Intervention Trial (PROBIT) Study Group. Breastfeeding and child cognitive development: new evidence from a large randomized trial. Arch. Gen. Psychiatry 2008, 65, 578-584.

8. Brenna, J.T.; Varamini, Bl.; Jensen, R.G.; Diersen-Schade, D.A.; Boettcher, J.A.; Arterburn, L.M. Docosahexaenoic and arachidonic acid concentrations in human breast milk worldwide. Am. J. Clin. Nutr. 2007, 85, 1457-1464.

9. Uylings, H.B.M. Development of the human cortex and the concept of 'critical' or 'sensitive' periods. Language Learning 2006, 56, 59-90.

10. De Graaf-Peters, V.B.; Hadders-Algra, M. Ontogeny of the human central nervous system: what is happening when? Early Hum. Dev. 2006, 82, 257-266.

11. Hadders-Algra, M. In Postural Control: A Key Issue in Developmental Disorders. Hadders-Algra, M., Brogren Carlberg, E., Eds.; Clin. Dev. Med. No. 179. Mac Keith Press: London, UK, 2008; pp. 22-73.

12. Krubitzer, L.; Kaas, J. The evolution of the neocortex in mammals: how is phenotypic diversity generated? Curr. Opin. Neurobiol. 2005, 15, 444-453.

13. Mrzljak, L.; Uylings, H.B.; Kostovic, I.; Vaneden, C.G. Prenatal development of neurons in the human prefrontal cortex. II. A quantitative Golgi study. J. Comp. Neurol. 1992, 316, 485-496.

14. Rakic, P. Pre- and post-developmental neurogenesis in primates. Clin. Neurosci. Res. 2002, 2, 29-39.

15. Miller, R.H. Regulation of oligodendrocyte development in the vertebrate CNS. Prog. Neurobiol. 2002, 67, 451-467.

16. Sowell, E.R.; Trauner, D.A.; Gamst, A.; Jernigan, T.L. Development of cortical and subcortical brain structures in childhood and adolescence: a structural MRI study. Dev. Med. Child. Neurol. 2002, 44, 4-16.

17. Rakic, S.; Zecevic, N. Programmed cell death in the developing human telencephalon. Eur. J. Neurosci. 2000, 12, 2721-2734.

18. Galea, M.P.; Darian-Smith, I. Postnatal maturation of the direct corticospinal projections in the macaque monkey. Cereb. Cortex 1995, 5, 518-540.

19. Eyre, J.A.; Taylor, J.P.; Villagra, F.; Smith, M.; Miller, S. Evidence of activity-dependent withdrawal of corticospinal projections during human development. Neurology 2001, 57, 1543- 1554.

20. Goda, Y.; Davis, G.W. Mechanisms of synapse assembly and disassembly. Neuron 2003, 40, 243- 264.

21. Prechtl, H.F.R. Continuity of Neural Functions Form Prenatal to Postnatal Life; Clin. Dev. Med. No. 94; Blackwell Scientific Publications: Oxford, UK, 1984.

22. Kostovic, I.; Judas M. Prolonged coexistence of transient and permanent circuitry elements in the developing cerebral cortex of fetuses and preterm infants. Dev. Med. Child Neurol. 2006, 48, 388-393. 
23. Hadders-Algra, M. Putative neural substrate of normal and abnormal general movements. Neurosci. Biobehav. Rev. 2007, 31, 1181-1190.

24. Hadders-Algra, M.; Prechtl, H.F.R. Developmental course of general movements in early infancy. I: Descriptive analysis of change in form. Early. Hum. Dev. 1992, 28, 201-214.

25. Hadders-Algra, M. Two distinct forms of minor neurological dysfunction: perspectives emerging from a review of data of the Groningen Perinatal Project. Dev. Med. Child Neurol. 2002, 44, 561-571.

26. Martinez, M. Tissue levels of polyunsaturated fatty acids during early human development. $J$. Pediatr. 1992, 120, S129-138.

27. Diau, G.Y.; Hsieh, A.; Sarkadi-Nagy, E.; Wijendran, V.; Nathanielsz, P.; Brenna, J.T. The influence of long chain polyunsaturate supplementation on docosahexaenoic acid and arachidonic acid in baboon neonate central nervous system. B.M.C. Medicine 2005, 3, 11.

28. Lauritzen, L.; Hansen, H.S.; Jørgensen, M.H.; Michaelsen, K.F. The essentiality of long chain n-3 fatty acids in relation to development and function of the brain and retina. Progr. Lipid Res. 2001, 40, 1-94.

29. Percy, P.; Percy, A.; Vilbersson, G.; Mansson, J-E. Polyunsaturated fatty acid accretion in first- and second-trimester human fetal brain: lack of correlation with levels in paired placental samples. Biochem. Mol. Med. 1996, 59, 38-43.

30. Clandinin, M.T.; Chapell, J.E.; Leong, S.; Heim, T.; Swyer, P.R.; Chance, P.W. Intrauterine fatty acid accretion rates in human brain: implications for fatty acid requirements. Early Hum. Dev. 1980, 4, 121-129.

31. Martinez, M.; Mougan, I. Fatty acid composition of human brain phospholipids during normal development. J. Neurochem. 1998, 71, 2528-2533.

32. Green, P.; Yavin, E. Mechanisms of docosahexaenoic acid accretion in the fetal brain. $J$. Neurosci. Res. 1998, 52, 129-136.

33. Larque, E.; Demmelmair, H.; Koletzko, B. Perinatal supply and metabolism of long-chain polyunsaturated fatty acids: importance for the early development of the nervous system. Ann. N. Y. Acad. Sci. 2002, 967, 299-310.

34. Simmer, K.; Patole, S.K.; Rao, S.C. Longchain polyunsaturated fatty acid supplementation in infants born at term. Cochrane Database Syst. Rev. 2008, 1, CD000376.

35. Uauy, R.; Hoffman, D.R.; Mena, P.; Llanos, A.; Birch, E.E. Term infant studies of DHA and ARA supplementation on neurodevelopment: results of randomized controlled trials. J. Pediatr. 2003, 143, S17-S25.

36. Hadders-Algra, M. The role of long-chain polyunsaturated fatty acids (LCPUFA) in growth and development. Adv. Exp. Med. Biol. 2005, 569, 80-94.

37. Carlson, S.E.; Ford, A.J.; Werkman, S.H.; Peeples, J.M.; Koo, W.K.K. Visual acuity and fatty acid status of term infants fed human milk and formulas with and without docosahexaeoate and arachidonate from egg yolk lecithin. Pediatr. Res. 1996, 39, 882-888.

38. Teller, D.Y.; McDonald, M.A.; Preston, K.; Sebris, S.L.; Dobson, V. Assessment of visual acuity in infants and children: the acuity card procedure. Dev. Med. Child Neurol. 1986, 28, 779-789. 
39. Auestad, N.; Montalto, B.; Hall, R.T.; Fitzgerald, K.M.; Wheeler, R.E.; Connor, W.E.; Neuringer, M.; Connor, S.L.; Taylor, J.A.; Hartmann, E.E. Visual acuity, erythrocyte fatty acid composition, and growth in term infants fed formulas with long chain polyunsaturated fatty acids for one year. Pediatr. Res. 1997, 341, 1-10.

40. Birch, E.E.; Hale, L.A. Criteria for monocular acuity deficit in infancy and early childhood. Invest. Ophthalmol. Vis. Sci. 1988, 29, 636-643.

41. Taylor, M.J.; McCulloch, D.L. Visual evoked potentials in infants and children. J. Clin. Neurophysiol. 1992, 9, 357-372.

42. Auestad, N.; Halter, R.; Hall, R.T.; Blatter, M.; Bogle, M.L.; Burks, W.; Erickson, J.R.; Fitzgerald, K.M.; Dobson, V.; Innis, S.M.; Singer, L.T.; Montalto, M.B.; Jacobs, J.R.; Qiu, W.; Bornstein, M.H. Growth and development in term infants fed long-chain polyunsaturated fatty acids: a double-masked randomized, parallel, prospective, multivariate study. Pediatrics 2001, 108, 372-381.

43. Agostoni, C.; Trojan, T.; Bellu, R.; Riva, E.; Giovannini, M. Neurodevelopmental quotient of healhty term infants at 4 months and feeding practice: the role of long-chain polyunsaturated fatty acids. Pediatr. Res. 1995, 38, 262-266.

44. Brunet, O.; Lézine, I. Le Developpement Psychologique De La Premiere Enfance, 2nd Edition; Presse Universitaire de France: Paris, France, 1966.

45. Bouwstra, H.; Dijck-Brouwer, D.A.J.; Wildeman, J.A.L.; Tjoonk, H.M.; Van der Heide, J.C.; Boersma, E.R.; Muskiet, F.A.J.; Hadders-Algra, M. Long-chain polyunsaturated fatty acids have a positive effect on the quality of general movements of healthy term infants. Am. J. Clin. Nutr. 2003, 78, 313-318.

46. Hadders-Algra, M. General movements: a window for early identification of children at high risk of developmental disorders. J. Pediatr. 2004, 145: S12-S18.

47. Makrides, M.; Neumann, M.A.; Simmer, K.; Gibson, R.A. A critical appraisal of the role of dietary long-chain polyunsaturated fatty acids on neural indices of term infants: a randomized, controlled trial. Pediatrics 2000, 105, 32-38.

48. Makrides, M.; Neumann, M.; Simmer, K.; Pater, J.; Gibson, R.A. Are long-chain polyunsaturated fatty acids essential nutrients in infancy? Lancet 1995, 345, 1463-1468.

49. Birch, E.E.; Hofman, D.R.; Uauy, R.; Birch, D.G.; Pastridge, C. Visual acuity and the essentiality of docosahexaenoic acid and arachidonic acid in the diet of term infants. Pediatr. Res. 1998, 44, 201-209.

50. Birch, E.E.; Castañeda, Y.S.; Wheaton, D.H.; Birch, D.G.; Uauy, R.D.; Hoffman, D.R. Visual maturation of term infants fed long-chain polyunsaturated fatty acid-supplemented or control formula for 12 mo. Am. J. Clin. Nutr. 2005, 81, 871-879.

51. Birch, E.E.; Morale, S.E.; Jeffrey, B.G.; O'Connor, A.R.; Fawcett, S.L. Measurement of stereoacuity outcomes at ages 1 to 24 months: Randot Stereocards. J. AAPOS 2005, 9, 31-36.

52. Ünay, B.; Sarici, Ü.; Ulas, Ü.H.; Akin, R.; Alpay, F.; Gökçay, E. Nutritional effects on auditory brainstem maturation in healthy term infants. Arch. Dis. Child Fetal Neonatal Ed. 2004, 89, S177-S179.

53. Wilkinson, A.R.; Jiang, Z.D. Brainstem auditory evoked response in neonatal neurology. Semin. Fetal Neonatal Med. 2006, 11, 444-451. 
54. Birch, E.E.; Carlson, S.E.; Hoffman, D.R.; Fitzgerald-Gustafson, K.M.; Fu, V.L.; Drover, J.R.; Castañeda, Y.S.; Minns, L.; Wheaton, D.K.; Mundy, D.; Marunycz, J.; Diersen-Schade, D.A. The DIAMOND (DHA Intake And Measurement Of Neural Development) Study: a double-masked, randomized controlled clinical trial of the maturation of infant visual acuity as a function of the dietary level of docosahexaenoic acid. Am. J. Clin. Nutr. 2010, 91, 848-859.

55. Scott, D.T.; Janowsky, J.S.; Carroll, R.E.; Taylor, J.A.; Auestad, N.; Montalto, M.B. Formula supplementation with long-chain polyunsaturated fatty acids: are there developmental benefits? Pediatrics 1998, 102, E59.

56. Bayley, N. Bayley Scales of Infant Development, 2nd ed.; Psychological Corporation: San Antonio, TX, USA, 1993.

57. Fenson, L.; Marchman, V.A.; Thal, D.; Dale, P.; Reznick, S.; Bates, E. The MacArthur Communicative Development Inventories: User's Guide and Technical Manual, 2nd Ed.; Paul. H. Brookes Publishing Co.: Baltimore, MD, USA, 2007.

58. Fagan, J.F.; Singer, L.T. In Advances in Infancy Research; Lipsitt, L.P., Ed; Ablex: Norwood, NJ, USA, 1983; Volume 2, pp. 31-72.

59. Rothbart, M.K. Measurement of temperament in infancy. Child. Dev. 1981, 52, 569-578.

60. Willats, P.; Forsyth, J.S.; Dimodugno, M.K.; Varma, S.; Colvin, M. Effect of long-chain polyunsaturated fatty acids in infant formula on problem solving at 10 months of age. Lancet 1998, 352, 688-691.

61. Auestad, N.; Scott, D.T.; Janowsky, J.S.; Jacobsen, C.; Caroll, R.E.; Montalto, M.B.; Halter, R.; Qui, W.; Jacobs, J.R.; Connor, W.E.; Connor, S.L.; Taylor, J.A.; Neuringer, M.; Fitzgerald, K.M.; Hall, R.T. Visual, cognitive and language assessments at 39 months: a follow-up study of children fed formulas containing long-chain polyunsaturated fatty acids to 1 year of age. Pediatrics 2003, 112, 177-1183.

62. Beery, K.E. Administration, Scoring and Teaching Manual for the Beery-Buktenica Developmental Test of Visual-motor Integration with Supplemental Developmental Tests of Visual Perception and Motor Coordination; Modern Curriculum Press: Toronto, NJ, USA, 1997.

63. Terman, L.M.; Merrill, M.A. Stanford-Binet Intelligence Scale Form L-M, 3rd Edition; Houghton Mifflin: Chicago, IL, USA, 1973.

64. Bouwstra, H.; Dijck-Brouwer, D.A.J.; Boehm, G.; Boersma, E.R.; Muskiet, F.A.J.; HaddersAlgra, M. Long-chain polyunsaturated fatty acids and neurological developmental outcome at 18 months in healthy term infants. Acta Paediatr. 2005, 94, 26-32.

65. Hempel, M.S. The Neurological Examination for Toddler-Age. PhD-thesis, University of Groningen, Groningen, The Netherlands, 1993.

66. Touwen, B.C.L. Examination of the Child with Minor Neurological Dysfunction, Clinics in Developmental Medicine No. 71; Mac Keith Press: London, UK, 1979.

67. Agostoni, C.; Trojan, S.; Bellu, R.; Riva, E.; Bruzzese, M.G.; Giovannini, M. Developmental quotient at 24 months and fatty acid composition of diet in early infancy: a follow-up study. Arch. Dis. Child. 1997, 76, 421-424.

68. Lucas, A.; Stafford, M.; Morley, R; Abbott, R.; Stephenson, T.; MacFayden, U.; Elias-Jones, A.; Clements, H. Efficacy and safety of long-chain polyunsaturated fatty acid supplementation of infant-formula milk: a randomised trial. Lancet 1999, 354, 1948-1954. 
69. Knobloch, H.; Passamanick, B.; Sherard, S. A developmental screening inventory for infants. Pediatrics 1966, 38, 1095-1108.

70. Singhal, A.; Morley, R.; Cole, T.J.; Kennedy, K.; Sonksen, P.; Isaacs, E.; Fewtrell, M.; Elias-Jones, A.; Stephenson, T.; Lucas, A. Infant nutrition and stereoacuity at age 4-6 y. Am. J. Clin. Nutr. 2007, 85, 152-159.

71. Sonksen, P.M.; Silver, J. The Sonksen-silver Visual Acuity System. Test System and Instruction Manual; Keeler: Windsor, UK, 1988.

72. Birch, E.E.; Garfield, S.; Hofman, D.R.; Uauy, R.; Birch, D.G. A randomized controlled trial of early dietary supply of long-chain polyunsaturated fatty acids and mental development in term infants. Dev. Med. Child Neurol. 2000, 42, 174-181.

73. Birch, E.E.; Garfield, S.; Castañeda, Y.; Hughbanks-Wheaton, D.; Uauy, R.; Hoffman, D. Visual acuity and cognitive outcomes at 4 years of age in a double-blind, randomized trial of long-chain polyunsaturated fatty acid-supplemented infant formula. Early Hum. Dev. 2007, 83, 279-284.

74. Moke, P.S.; Turpin, A.H.; Beck, R.W.; Holmes, J.M.; Repka, M.X.; Birch, E.E.; Hertle, R.W.; Kraker, R.T.; Miller, J.M.; Johnson, C.A. Computerized method of visual acuity testing: adaptation of the Amblyopia Treatment Study visual acuity testing protocol for children. Am. J. Ophthalmol. 2001, 132, 903-909.

75. Wechsler, D. Wechsler Preschool and Primary Scale of Intelligence-Revised; Psychological Corporation: London, UK, 1989.

76. Lanting, C.I.; Patandin, S.; Weisglas-Kuperus, N.; Touwen, B.C.; Boersma, E.R. Breastfeeding and neurological outcome at 42 months. Acta Paediatr. 1998, 87, 1224-1229.

(C) 2010 by the authors; licensee MDPI, Basel, Switzerland. This article is an Open Access article distributed under the terms and conditions of the Creative Commons Attribution license (http://creativecommons.org/licenses/by/3.0/). 